\title{
WESTAD, Odd Arne. - The Global Cold War : Third World Interventions and the Making of Our Times
}

\section{Patrice Yengo}

\section{OpenEdition}

\section{Journals}

Édition électronique

URL : http://journals.openedition.org/etudesafricaines/20797

DOI : 10.4000/etudesafricaines.20797

ISSN : 1777-5353

\section{Éditeur}

Éditions de l'EHESS

\section{Édition imprimée}

Date de publication : 1 juillet 2017

Pagination : 478-481

ISBN : 978-2-7132-2688-5

ISSN : 0008-0055

\section{Référence électronique}

Patrice Yengo, «WESTAD, Odd Arne. - The Global Cold War : Third World Interventions and the Making of Our Times », Cahiers d'études africaines [En ligne], 226 | 2017, mis en ligne le 01 avril 2017, consulté le 24 septembre 2020. URL : http://journals.openedition.org/etudesafricaines/20797 ; DOI : https:// doi.org/10.4000/etudesafricaines.20797

Ce document a été généré automatiquement le 24 septembre 2020.

(c) Cahiers d'Études africaines 


\title{
WESTAD, Odd Arne. - The Global Cold War : Third World Interventions and the Making of Our Times
}

\author{
Patrice Yengo
}

\section{RÉFÉRENCE}

WESTAD, Odd Arne. - The Global Cold War : Third World Interventions and the Making of Our Times. New York, Cambridge University Press, 2005, 484 p., bibl.

1 À la chute du mur de Berlin, le sort du monde semblait scellé : la démocratie et le libéralisme avaient triomphé et leur extension à l'ensemble de la planète, qu'augurait la globalisation de l'économie, n'était plus qu'une question de temps. Le livre de Francis Fukuyama, La Fin de l'Histoire ${ }^{32}$, a donné de cette thèse son corpus théorique le plus abouti. Selon cet auteur, la période ouverte par l'effondrement du « communisme » est celle d'une «fin de l'Histoire », entendue comme clôture de nouveaux modèles sociaux, politiques ou économiques et victoire de l'idée de la démocratie libérale, c'est-à-dire d'une société basée sur les valeurs de liberté et d'égalité que de plus en plus de sociétés adopteront comme modèle. Ces prédictions ont été déjouées par l'histoire qui a suivi, mais le matériau qui leur a donné naissance n'a eu de cesse de nourrir une immense littérature historiographique et (géo)politique sur les stratégies et tactiques des deux superpuissances dans leurs confrontations à travers le monde. On peut classer les études qui en découlent, au-delà de leur diversité interne, en deux grands courants. Un premier courant axé sur la bipolarisation du monde et le conflit Est-Ouest qui a dominé le monde depuis les accords de Yalta ${ }^{33}$, et un second qui approche l'histoire mondiale sur la longue durée, permettant d'en déduire les événements actuels de notre planète ${ }^{34}$. 
Odd Arne Westad, directeur du Cold War Studies Centre de la London School of Economics and Political Science, se situe dans le renouveau de l'historiographie de la Guerre froide ayant élargi considérablement le cadre d'analyse de la conflictualité EstOuest depuis l'ouverture aux chercheurs de nouvelles archives de l'URSS qu'il a longuement consultées en même temps qu'il a recueilli de nombreux témoignages d'hommes de terrain. S'il peut être rattaché peu ou prou à l'un ou l'autre de ces courants, il s'en démarque ostensiblement par sa capacité de décentrer le regard à partir de ce que l'on appelait la "périphérie » du système-monde. De la prolifération nucléaire au terrorisme international à l'échelle mondiale, les questions de politique internationale restent certes encore tributaires des prérogatives de la Guerre froide, mais, selon Westad, c'est la confrontation des États-Unis et de l'Union soviétique dans le Tiers-monde qui en explique les pernicieux héritages actuels. Ce qu'il réaffirme lors d'une table-ronde consacrée à son livre: «Ce que j'ai essayé de faire, c'est plutôt d'amener le Tiers-monde sur la scène, pour rectifier l'équilibre [...] dans l'historiographie à son sujet. Pendant trop longtemps, l'Europe a été perçue non seulement comme la seule cause de la Guerre froide, mais aussi comme son moteur. L'existence d'une vue d'ensemble du conflit dans le Tiers-monde aidera, je l'espère, ceux qui travaillent principalement en Europe à ne pas seulement voir des influences et des parallèles qu'ils n'avaient pas vus auparavant, mais aussi à repenser la Guerre froide comme un système global $\aleph^{35}$. C'est cette globalité qui imprègne chaque parcelle de la conflictualité et détermine l'action des acteurs sur le terrain. C'est aussi cette globalité qui permet à Westad de s'affranchir des présupposés systémiques qui semblaient opposer les deux superpuissances pour relever que leurs visées prenaient leur source historique dans l'idéologie du progrès et de la modernité des Lumières au nom de laquelle ils avaient uni leurs efforts pour revendiquer l'indépendance des anciennes colonies britanniques et françaises. Ce qui pousse l'auteur à en examiner d'emblée les fondements. Dans cette vaste étude qui synthétise une grande partie de la littérature volumineuse sur l'histoire internationale de la Guerre froide et introduit une variété de sources d'archives nouvellement disponibles, le Tiers-monde n'apparaît plus simplement comme une arène où se testait la supériorité de chaque bloc mais comme le terrain privilégié d'un affrontement idéologique sans partage.

3 En partant de l'exploration historique de l'attitude des dirigeants américains, soviétiques et du Tiers-monde dans le monde de la Guerre froide, les trois premiers chapitres nous introduisent au cœur de son propos. Ils sont, de ce fait, entièrement consacrés à l'analyse des présupposés idéologiques qui ont servi de trame aux interventions - fort coûteuses au demeurant - des deux superpuissances dans le Tiers-monde. Et Westad d'en conclure que pour les États-Unis, la primauté revenait à la défense de la liberté tandis que la lutte pour la justice servait d'argument aux Soviétiques; deux dogmes universalistes qui, pour être à la base de la création des États-Unis et de l'Union soviétique, n'en avaient pas moins concrètement pour nom, libéralisme économique dans le premier cas et socialisme dans le second. Westad bat ainsi en brèche le formalisme de certains historiens qui voudraient que l'idéologie ne relève que de la théorie politique, ce qui reviendrait à dire que seuls les Soviétiques auraient eu une idéologie - le marxisme-léninisme dont ils se revendiquaient ouvertement - et que les USA en auraient été dépourvus. S'insurgeant contre certains courants du révisionnisme historique qui fait de l'expansionnisme soviétique la première, sinon l'unique cause de l'affrontement Est-Ouest, il rappelle que l'interventionnisme des États-Unis ne date pas de la Guerre froide. 
4 En effet, bien que les USA soient nés d'une lutte anti-impérialiste, ils ne se sont pas privés, à la suite de leur victoire dans la guerre hispano-américaine en 1898, d'intervenir en "empire de la liberté », notamment dans les affaires des peuples non blancs (Cuba, Philippines, Porto-Rico, Hawaï, Haïti). De la même façon, l'idéologie de la Guerre froide soviétique reliait, au-delà de la théorie stalinienne de la construction du socialisme dans un seul pays, les revendications modernistes et universalistes du marxisme au projet expansionniste des tsars hérité du XIX siècle.

5 Les similitudes entre les deux camps apparaissent ici clairement. Les deux parties se sont projetées dans une mission historique dont l'horizon était balisé par le discours de la modernité (rationalité, progrès, science, justice et liberté). C'est à l'aune de cette compétition mimétique des universalismes, pour reprendre un mot de René Girard, qu'il faut appréhender leur hostilité croissante, notamment aux premières années de la Guerre froide.

6 Cela n'empêche pas que cette idéologie se nourrisse de ses contradictions et que la pratique de terrain se trouve quelquefois en divergence avec cette même idéologie. Du moins en apparence. Ainsi Staline a signé des accords avec Tchang Kaï-chek aux dépens de Mao Zedong en 1945. Loin d'être contre-nature, ces accords relevaient de l'idéologie de la Révolution par étapes qui postulait que la Chine n'était pas mûre, à ce stade de son développement, pour la révolution socialiste et que les communistes devaient se ranger derrière le Kuomintang. De la même façon en 1977, Jimmy Carter, préférant le Shah aux démocrates iraniens, montrait que Mohammad Reza Pahlavi était le meilleur garant de la modernisation de l'Iran. On peut penser avec Westad que c'est cette même idéologie qui alimentait les luttes intestines des bureaucraties locales qui devaient répondre aux interventions des superpuissances tout en naviguant entre les exigences des fractions concurrentes et les populations en quête de changement.

7 Ce sont ainsi deux dogmes de la modernité que les États-Unis et l'Union soviétique ont représentés pour les pays du Tiers-monde. Il se trouve que l'intervention injonctive au nom de ces deux dogmes à prétention universelle a fini par apparaître pour les peuples du Tiers-monde comme le prolongement des politiques coloniales des États européens qui, elles aussi, se revendiquaient d'une mission civilisatrice. Vue sous cet angle, la Guerre froide n'était plus simplement une opposition entre l'Est et l'Ouest mais bien une coalition objective Est-Ouest contre le Sud.

8 L'analyse des origines idéologiques de la politique américaine et soviétique de la Guerre froide, ouvre à Westad le champ de la décolonisation qu'il aborde comme une donne géopolitique inédite ayant permis aux dirigeants des États nouvellement indépendants de se positionner sur l'échiquier international pour leurs plans de développement, laissant aux superpuissances l'occasion de prouver la fiabilité de leur modèle ou d'étendre leur influence. L'Inde et l'Indonésie sont l'illustration parfaite de la manière dont certains dirigeants ont su capitaliser les contradictions de la Guerre froide. En restant non alignés et en jouant les superpuissances les unes contre les autres, de nombreux nouveaux États ont été en mesure de disposer d'une certaine indépendance dans un monde de plus en plus bipolarisé et où le Tiers-monde comme entité a été unifié sinon créé par cet interventionnisme incessant (chapitre 4).

9 Partant de ce cadre conceptuel, Westad aboutit dans le chapitre 5 aux conséquences de la révolution cubaine et de la guerre du Vietnam, deux défis majeurs auxquels ont été confrontés les États-Unis pour maintenir leur hégémonie. Mais c'est dans les chapitres suivants, relatifs aux interventions de la Guerre froide en Afrique dans les années 1960 
et 1970 qui nous intéressent particulièrement, que se révèle l'originalité de l'ouvrage de Westad. Car c'est en Afrique du Sud, en Angola, en Éthiopie, au Zaïre et en GuinéeBissau que la Guerre froide opère un tournant dont les conséquences restent encore identifiables sur le développement de ce continent, et ce qui se joue dans ces pays n'est pas moins que le sort de la confrontation entre les deux blocs. Malgré les tentatives infructueuses de l'URSS d'implanter le socialisme dans la corne de l'Afrique, Moscou et La Havane signent une victoire concrète. Ils ont réussi à promouvoir des alliances avec les dirigeants anticolonialistes, désenchantés par la brutalité du modèle américain. Toutefois, la montée de l'islamisme radical en Iran et en Afghanistan à la fin des années 1970 et le défi qu'il pose à partir des années 1980, décrits aux chapitres 8 et 9 , viennent rétablir un équilibre au profit des États-Unis qui sonne le glas de l'interventionnisme de l'Union soviétique dont les coûts économiques, politiques et humains participent dans une certaine mesure aux causes fondamentales de sa désintégration.

Comment résumer un livre d'une telle richesse? Ce qui fait d'abord l'intérêt de cet ouvrage et qui d'emblée le rend passionnant, c'est l'ambition d'O. A. Westad d'offrir une nouvelle version de l'histoire de la Guerre froide en prenant pour point de départ, non les relations entre les deux superpuissances, mais leurs rapports avec le Tiersmonde. Cette ambition se trouve ici exaucée grâce à cette approche méthodologique singulière qui replace le concept d'idéologie au cœur de son travail. Elle permet en effet de comprendre pourquoi certaines interventions ont été poursuivies en dépit du bon sens jusqu'à leur point de rupture, faisant de la Guerre froide une tragédie sans précédent pour les pays du Sud. Nous sommes loin du triomphalisme américain qui considère que la fin de la Guerre froide est une victoire des États-Unis car, ainsi que Westad le mentionne en conclusion : «Seen from a Third World perspective, the results of America's interventions are truly dismal. Instead of being a force for good [...] these incursions have devastated many societies and left them more vulnerable to further disasters of their own making ( (p. 404). L'axe du mal n'aura finalement été «vaincu » qu'au prix d'un reniement constant de ses propres idéaux et d'un désastre sans équivalent des sociétés des jeunes nations.

11 Pour tous ceux qui s'intéressent à l'histoire de la Guerre froide du point de vue des pays $\mathrm{du}$ Tiers-monde, The Global Cold War est une œuvre remarquable par son souffle géographique et historique qui réinvestit ce passé récent d'un regard neuf. Étayée par un index riche de nombreuses entrées et des cartes d'une grande précision, elle procède aussi d'une démarche pédagogique, très utile en ces temps d'obscurantisme décomplexé. Mais cela supposait un travail considérable de recherche dans les archives soviétiques et l'utilisation des documents américains récemment déclassifiés sans oublier les apports massifs des sources primaires allemandes, britanniques, sudafricaines, chinoises et autres. Travail dont Odd Arne Westad s'est bien acquitté car, bien que cet ouvrage ait déjà une douzaine d'années, son actualité est loin d'être démentie. 


\section{NOTES}

32. F. FUKUYAMA, La fin de l'histoire et le dernier homme, Paris, Flammarion, 1992.

33. A. CONTE, Yalta ou le partage du monde, Paris, R. Laffont, 2001 ; N. FRIEDMAN (ed.), The Cold War Experience : 1945-1991, London, Carlton Books Ltd, 2005 ; S. JEANNESSON, La Guerre froide, Paris, La Découverte, 2014 ; G.-H. SouTou, La Guerre froide 1943-1990, Paris, Pluriel, 2011.

34. A. FonTAINe, La Guerre froide, 1917-1991, Paris, Éditions du Seuil, 2006. À ces deux courants, il faut peut-être joindre un autre plus prospectif, dont le grand représentant est S. Huntington, Le Choc des civilisations, Paris, Odile Jacob, 2014 [1997], qui, partant des mêmes prémisses, anticipe sur un avenir où la politique internationale ne serait plus le fait d'oppositions idéologiques, comme ce fut le cas entre les deux blocs de la Guerre froide. Les conflits du monde engageraient désormais de grandes civilisations, regroupées par affinités culturelles. Dans cette nouvelle configuration, la civilisation occidentale se trouve confrontée, cette fois-ci, à la puissance grandissante de l'islam et à la montée en force de l'économie chinoise.

35. T. MADDUX (ed.), « H-Diplo Roundtable Reviews », 8, 12 (2007), https://issforum.org/ roundtables/PDF/GlobalColdWar-Roundtable.pdf. Parmi les participants à la table ronde : Jerald Combs, William Hitchcock, David Painter, Natalia Yegorova. 